Review Article

\title{
Current Evidence Does Support the Use of KT to Treat Chronic Knee Pain in Short Term: A Systematic Review and Meta-Analysis
}

\author{
Wen-hao Luo ${ }^{1}$ and Ye Li ${ }^{2}$ \\ ${ }^{1}$ Department of General Surgery, Peking Union Medical College Hospital, \\ Chinese Academy of Medical Sciences and Peking Union Medical College, Beijing 100730, China \\ ${ }^{2}$ Department of Orthopedics Surgery, Peking Union Medical College Hospital, \\ Chinese Academy of Medical Sciences and Peking Union Medical College, Beijing 100730, China \\ Correspondence should be addressed to Ye Li; liye@pumch.cn
}

Received 25 January 2021; Revised 8 March 2021; Accepted 13 March 2021; Published 23 March 2021

Academic Editor: Jian-Guo Zhou

Copyright (@) 2021 Wen-hao Luo and Ye Li. This is an open access article distributed under the Creative Commons Attribution License, which permits unrestricted use, distribution, and reproduction in any medium, provided the original work is properly cited.

Objective. To demonstrate whether KT is better than placebo taping, nonelastic taping, or no taping in reducing pain. Methods. PubMed, Embase, Web of Science, the Cochrane Central Library, and ClinicalTrials.gov were systematically searched up to 20 October 2020 for randomized controlled studies that used KT to treat chronic knee pain according to PRISMA guidelines. We extracted the mean differences and SD in pretreatment and posttreatment for selected outcomes measured in the experimental and control groups for subsequent meta-analyses. Results. In total, 8 studies involving 416 participants fulfilled the inclusion criteria. Our results indicated that KT is better than other tapings (placebo taping or nonelastic taping) in the early four weeks. The mean difference was -1.44 (95\% CI: $\left.-2.04--0.84, I^{2}=49 \%, P \leq 0.01\right)$. Treatment methods which were performed for more than six weeks $\left(0.16\right.$ ( $95 \%$ CI: $\left.-0.35-0.68, I^{2}=0 \%, P=0.53\right)$ ) show no significant difference in reducing pain. In studies in which visual analogue scale was measured, a positive effect was observed for KT combined with exercise program training $(-3.27$ (95\% CI: $\left.-3.69-2.85, I^{2}=0 \%, P<0.05\right)$ ). Conclusion. KT exhibited significant but temporary pain reduction.

\section{Introduction}

Chronic knee pain is an essential cause of pain, disabilities, and reductions in people's quality of life [1]. Chronic knee pain is created by chronic diseases which are commonly knee osteoarthritis $(\mathrm{OA})$ [2], patellofemoral pain syndrome $[3,4]$, after anterior cruciate ligament reconstruction [5], and pes anserinus tendinitis [6]. Surgery may provide significant therapy effects, but they are not widespread because of the risk of complications in operation and anesthesia. Consequently, the nonoperative measures are an optimal option for most patients with chronic knee pain, such as functional motion, reducing weight, pain killers, and corticosteroid injections. However, current evidence did not prove which one of those nonoperative therapies should be recommended as the first-line therapy based on comprehensive and individualized consideration of the adverse impacts and positive outcomes of patients [7]. Complementary therapy such as ginger in the management of chronic knee pain has been fully discussed in recent studies $[8,9]$.

Recently, KT has been applied in treating chronic knee pain as a novel nonoperative therapy [10]. Unfortunately, the positive outcome of KT in reducing chronic knee pain is still in dispute [10]. Many studies [11, 12] failed to prove the effectiveness of KT for chronic knee pain in clinical practice. Some studies [13-15] have proved that KT did reduce chronic knee pain, but there is no significant difference compared with other nonoperative therapies. At present, KT is mainly used to improve athletes' short-term muscle pain to improve athletic performance, but the effect of KT on reducing chronic pain caused by some chronic degenerative diseases is uncertain. Admittedly, there was no review of KT for chronic knee pain and no review proved the KT has 
a superior advantage in pain reduction compared with other approaches. With increasing clinical trials being carried out to evaluate the beneficial effects of KT for chronic knee pain, we grasped this opportunity to conduct a meta-analysis exploring the function of KT. Therefore, the aim of this article was to examine and summarize the evidence of recent RCTs regarding the effectiveness of KT [11-26]. We hypothesized that KT would result in significant pain reduction in early 4 weeks but no significant pain reduction after 4 weeks, which is the focused clinical question in this systematic review. However, there are some uncertainties and conflicts that underlie the hypotheticals. First of all, the relationship between KT application techniques and pain reduction remains controversial. Secondly, whether such pain relief is self-recovery of body function or the true function of KT is still uncertain. Last but not least, does the short-term effect of KT on chronic knee pain patients have its clinical significance? The answer is debatable. It is exceedingly important to figure out those hidden mysteries and help those individuals who believe the long-term benefits of KT get out of their misunderstandings. Therefore, we conducted a systematic review and meta-analysis to test this hypothesis.

\section{Methods}

2.1. Search Strategy. Relevant studies were searched and identified by individually searching the following databases: PubMed, Embase, Web of Science, the Cochrane Central Library, and ClinicalTrials.gov up to 20 October 2020 according to PRISMA guidelines. For all databases including grey literatures, reference list of related systematic reviews, and other related studies, the following search strategy was used with database-specific truncation terms: knee AND (tape OR Taping) AND pain (((osteoarthritis) OR (degenerate)) AND ((tape) OR (taping) OR (kinesiotape)) AND ((knee) OR (patellofemoral) OR (tibofemoral)) AND Clinical Trial [ptyp]. Eligibility assessment was performed by LUO Wen-Hao. Disagreements between reviewers were resolved by group discussion and consensus.

2.2. Inclusion and Exclusion Criteria. The protocol was established a priori. Eligibility was assessed by 2 independent reviewers (LUO Wen-Hao and LI Ye), with the consensus reached by discussing conflicts with a third investigator (Ruoyu Ji). Assessments were performed and repeated twice. Firstly, titles and abstracts were assessed. Then potentially qualified studies were obtained in full text and assessed through the PRISMA flow diagram chart [27] (Figure 1). All authors were familiar with the authorship of studies. There were no restrictions on the history of knee pain, nor on follow-up duration or taping times. In order to evaluate the combined effects of KT with other concurrent interventions, we included those relevant studies. Non-English studies were excluded.

Inclusion criteria are presented in Box 1.

Box 1. Inclusion criteria

Design
Randomised controlled trials

Publication languages

English only

Publication year

Up to October 2020

Patients

Adults

People with chronic knee pain diagnosed with knee OA, patellofemoral pain syndrome, pes anserinus tendinitis, or after anterior cruciate ligament reconstruction

Intervention

KT method

Outcome measure

Chronic knee pain

2.3. Data Collection and Analysis. Data such as authors, publication year, baseline information of participants, dropout rates, assessment time, and the outcome of the KT group and the control group (i.e., placebo taping, no taping, or other intervention) were extracted from each included trial.

2.4. Assessment of Study Quality. The methodologic quality of selected studies was blindly evaluated by 2 independent reviewers (LUO Wen-Hao and Li Ye). Disagreements between LUO Wen-Hao and Li Ye were discussed by the group and resolved by a third assessor. Quality was assessed using the Consort 2010 statement as well as Cochrane Library Scale.

2.5. Data Synthesis. The primary outcome of this metaanalysis is the comparison of short-term effect and longterm effect of $\mathrm{KT}$ in pain reduction. The secondary outcome is the comparison between Kinesio Taping combined with exercise program, and exercise program only comes to the secondary outcome. Pain scores (outcome) were transformed into percentages of the maximum possible score and reported as centimeters on a $10 \mathrm{~cm}$ analog scale, using the pain visual analogue scale (VAS), a standardized 11-point scale with a score of 10 being the most painful. The mean difference and standard deviation between $\mathrm{KT}$ and other interventions were determined. Effect sizes for KT and other interventions were derived by dividing the mean differences by the pooled SD. Data were entered in Cochrane Collaboration's Review Manager program (RevMan version 5.3, Cochrane Collaboration, Oxford, UK). We analyzed the standardized mean differences and 95\% CIs and performed tests of heterogeneity $\left(I^{2}\right)$ for outcomes. Fixed-effects or random-effects models were used appropriately and accordingly. The heterogeneity quantity was used to test heterogeneity between RCTs in our analysis. Moderate-tohigh heterogeneity $\left(I^{2}=50 \%\right)$ was explored using sensitivity analyses. 


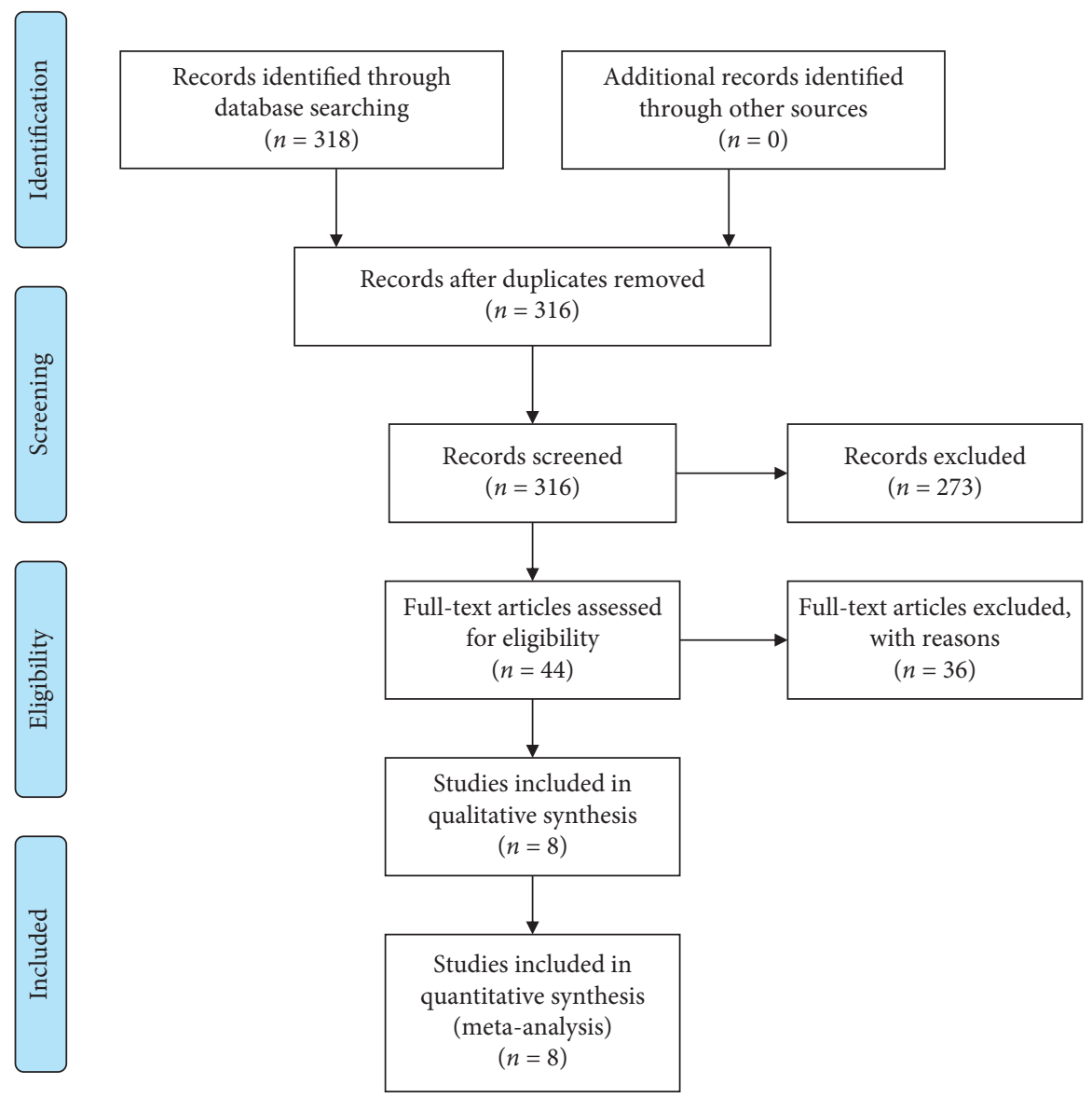

Figure 1: Inclusion and exclusion chart.

\section{Results}

3.1. Study Selection. 318 unique articles were selected, of which 8 fulfilled the inclusion criteria (Figure 1). The retrieved data of the 8 eligible studies are listed in Table 1.

3.2. Data Analysis. 8 studies investigated the effects of KT in pain reduction. Those studies in which the measurement has been performed for less than 4 weeks ( 5 studies, 202 participants) showed a mean difference in pain reduction of -1.44 (95\% CI: $-2.04-0.84, I^{2}=49 \%, P \leq 0.01$ ) (Figure 2). 3 studies (2012.Kuru T, 2017.chan MC, 2011.Akbas E, 132 participants) in which the treatment methods have been performed for 6 weeks showed no obvious difference in pain reduction $\left(0.16\left(95 \% \mathrm{CI}:-0.35-0.68, \mathrm{I}^{2}=0 \%, P=0.53\right)\right.$ ) (Figure 2). KT combined with exercise program decreased pain significantly compared with exercise program only (3 studies, 91 participants) $(-3.27 \mathrm{~cm}$ (95\% CI: $-3.69--2.85$; $\left.P \leq 0.01 ; I^{2}=0 \%\right)$ ) (Figure 3 ).

\section{Discussion}

This meta-analysis is the first study to put up with the temporary effects of KT on chronic knee pain relief compared with other taping methods. With the current evidences, we suggest KT temporarily controls the symptoms of chronic knee pain and KT should be applied with exercise program in relieving knee pain.

The underlying mechanism of chronic pain reduction in reference to KT remains unknown. The mechanism of its pain relief function is $\mathrm{KT}$ can enhance the spaces between skin and muscle by dragging skin, which as a result relieves local pressure and helps to accelerate circulation and promote lymphatic drainage [28]. Therefore, it can reduce pain, attenuate swelling, and alleviate muscle spasm [29]. Moreover, KT increases pressure on the knee joint which may constrict knee joint movement, improve joint stability and functional performance, and thus temporarily minimize the chance of joint injuries.

In terms of the results, we need to clarify the cause of chronic knee pain, whether it is due to aseptic inflammation caused by exercise, trauma, or strain, or OA caused by the wear and regression of articular cartilage. The pain caused by aseptic inflammation can be well alleviated through KT because the inflammation and pain are often transient, so the short-term effect will be better. But for some patients with OA or joint degeneration, KT cannot turn the worn cartilage into a new one, or repair it; therefore, that is why it cannot achieve the purpose of the long-term application. In addition, in terms of comfort and convenience, KT can be used for up to 7 days in chronic exercise pain because 7 days of 


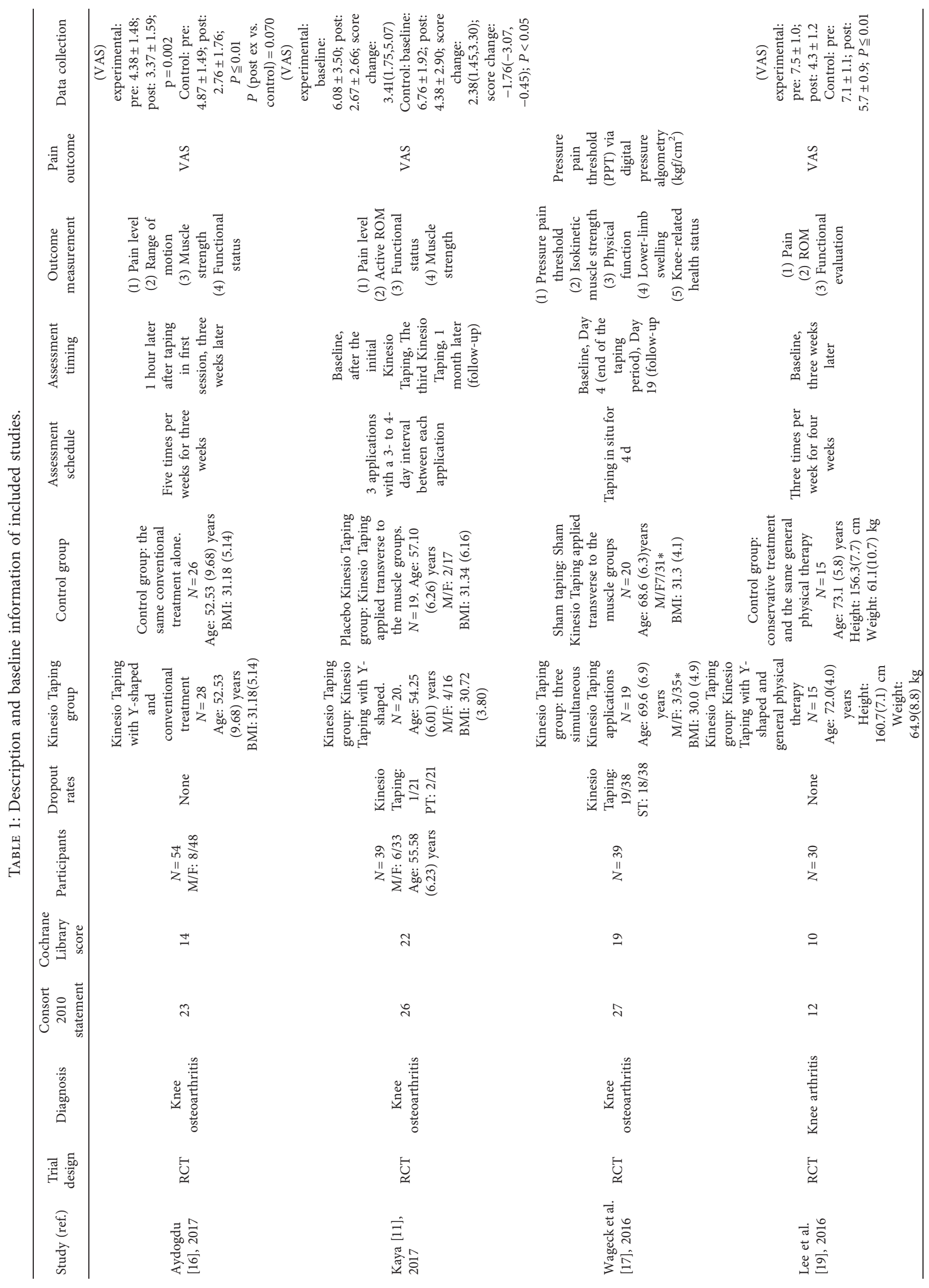




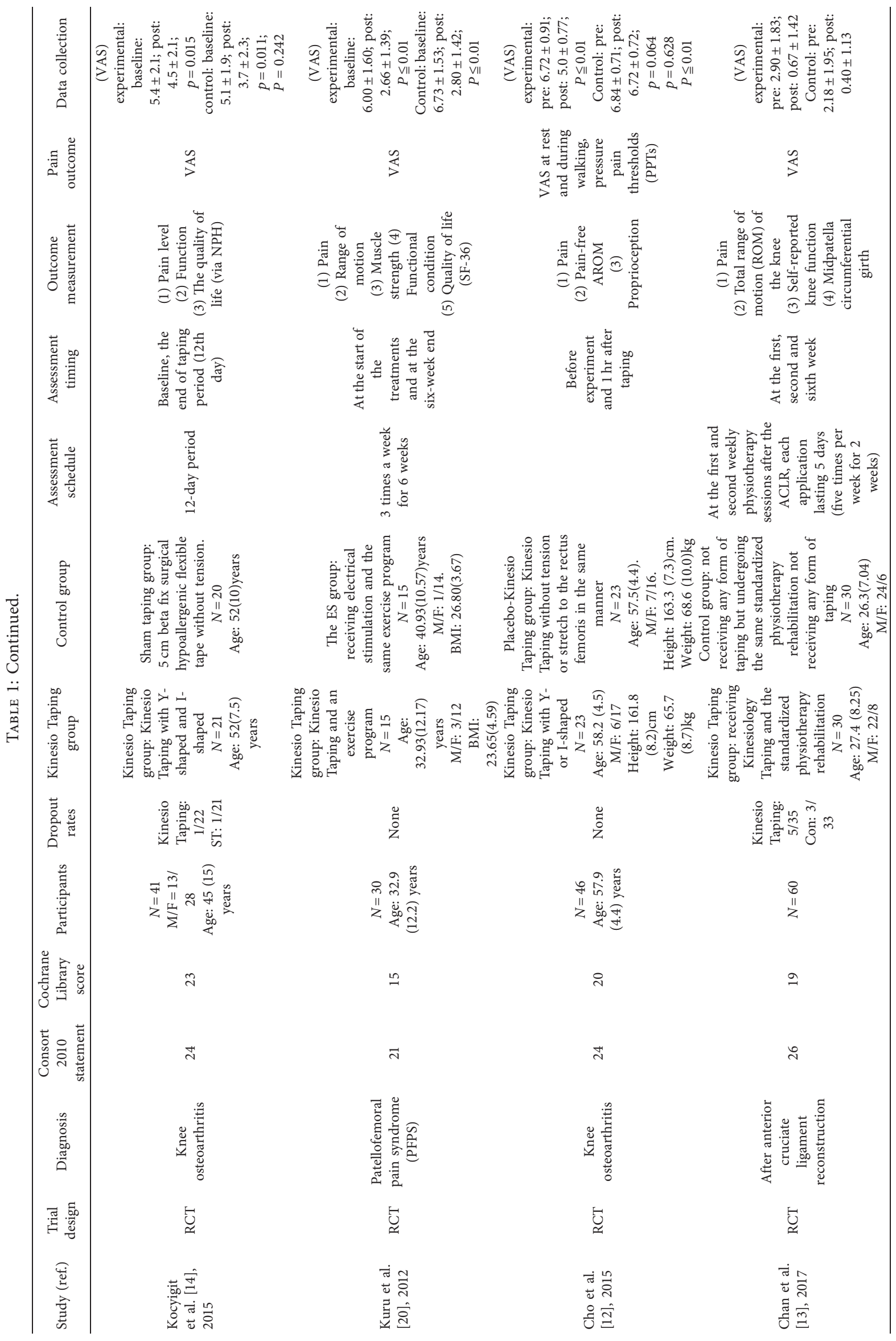




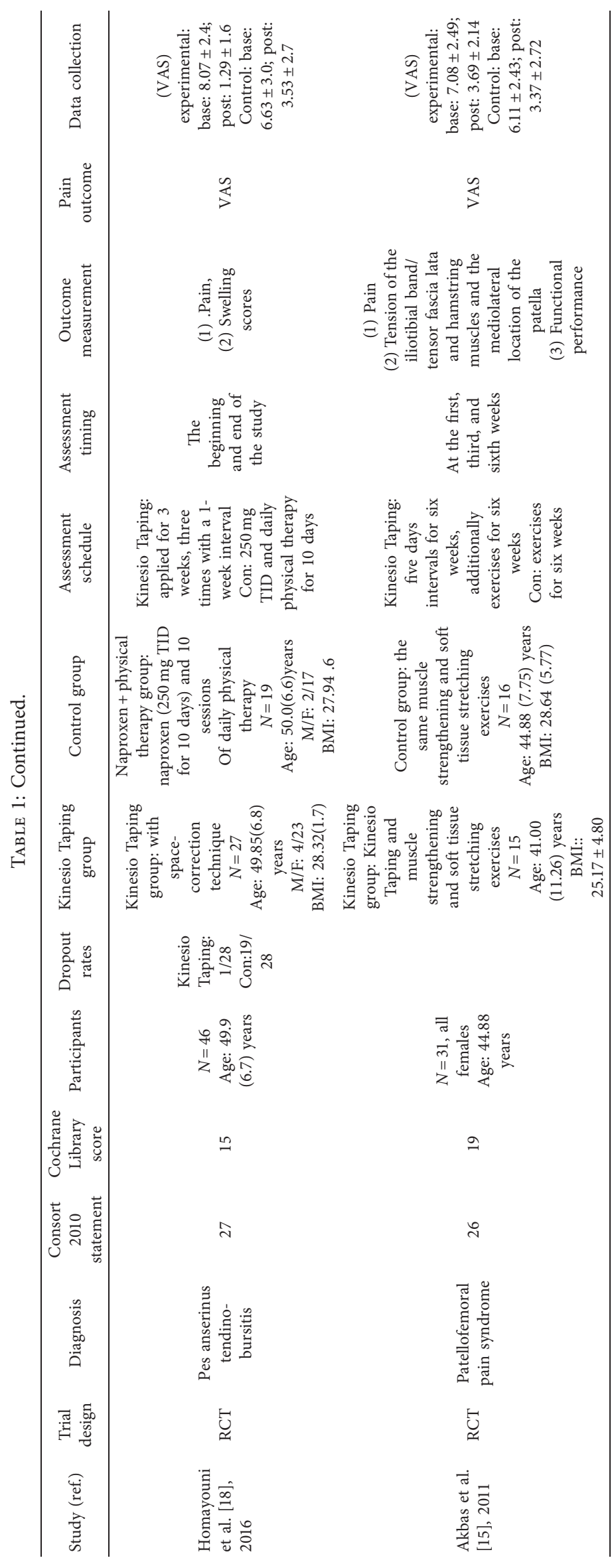




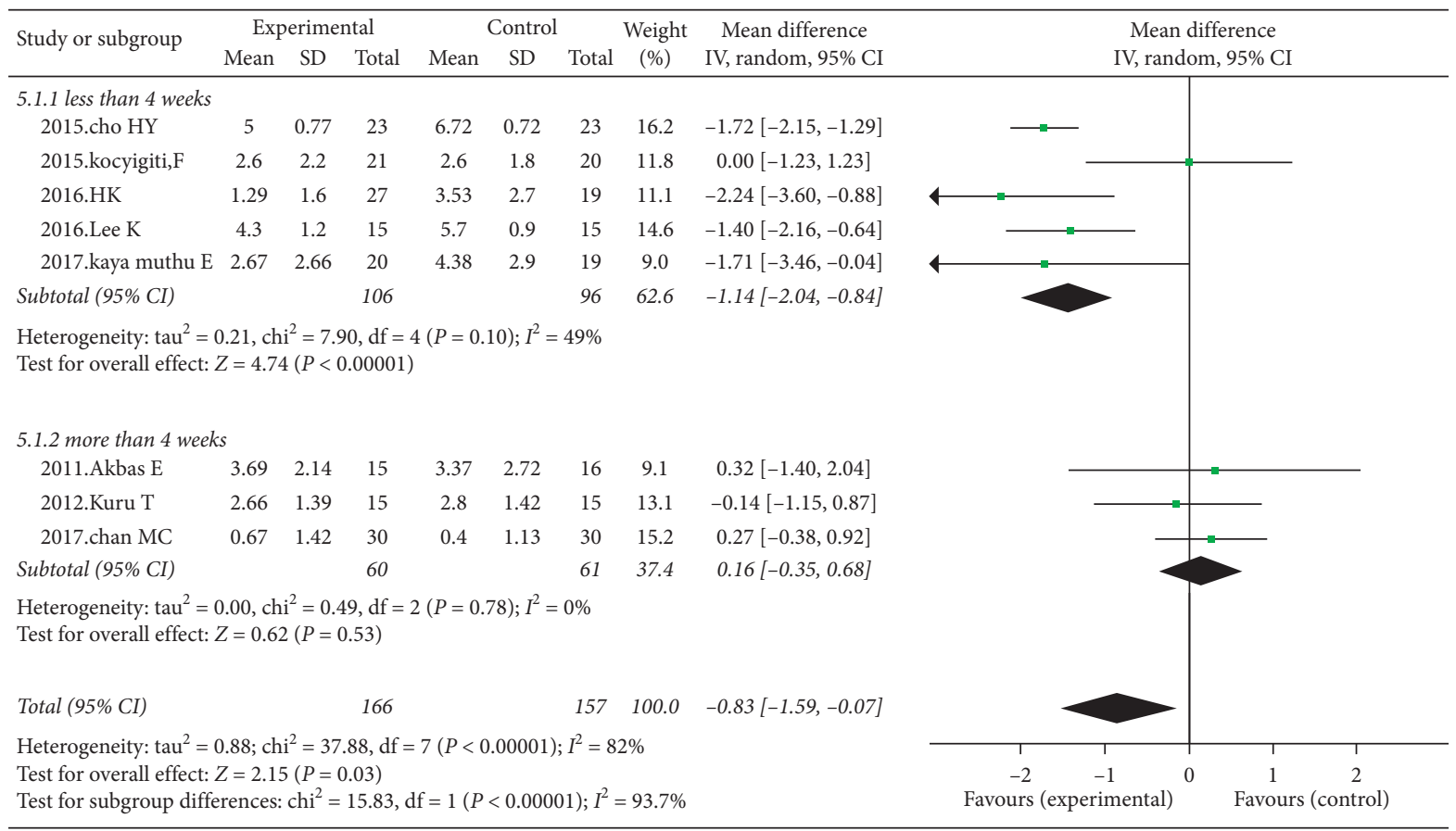

FIGURE 2: Effects of Kinesio Taping on chronic knee pain. Subgroup analysis: those studies in which the measurement was performed at less than 4 weeks are compared with the remaining 3 studies in which the assessments were performed at the six-week end in pain reduction.

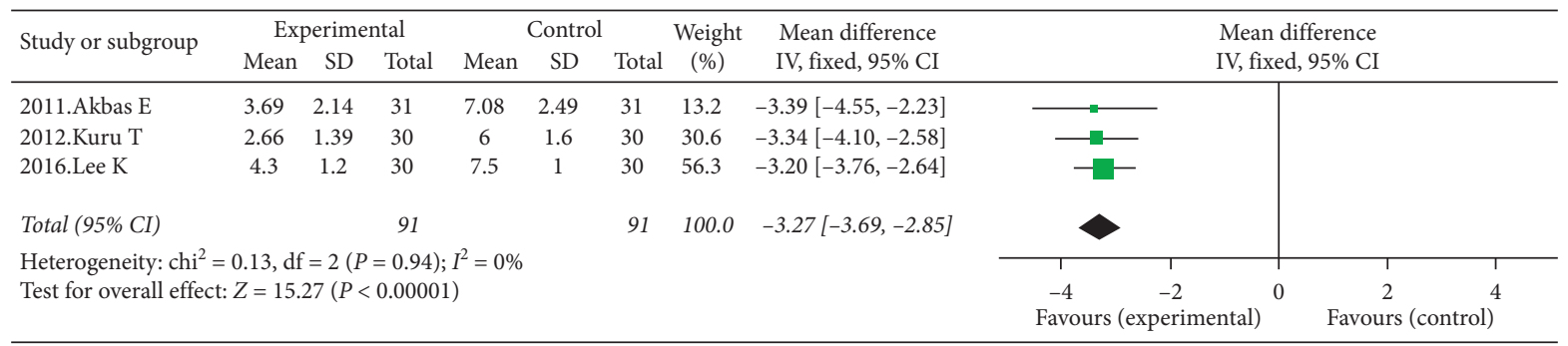

FIgURE 3: Effects of Kinesio Taping on chronic knee pain. Kinesio Taping combined with exercise program compared with exercise program only.

rehabilitation is sufficient for exercise-induced transient injury. But for chronic knee pain caused by OA with more than one month or two months, KT is not of great significance. KT has been used to improve lymphatic circulation and strengthen joint stability, but it does not eliminate inflammation, nourish cartilage, or even strengthen the surrounding muscles. Therefore, $\mathrm{KT}$ is effective for temporary treatment and cannot be used as a long-term treatment.

For the application of $\mathrm{KT}$ in patients with $\mathrm{OA}$, a large part of our treatment emphasizes the basic treatment, including weight control and strengthening of the surrounding muscles. During our basic treatment, some patients feel knee pain because of inappropriate exercise or excessive exercise. Then we can use KT to restrict the excessive exercise of the knee. Its benefits will be more obvious than nonsteroidal anti-inflammatory drugs, with smaller side effects. However, for some people with moderate or severe knee degeneration, the effect of KT will not be very good because KT cannot save cartilage wear back.
Overall, KT is a relatively cost-effective treatment intervention for chronic knee pain. It is commonly used as a sports and rehabilitation programme. However, current evidences from clinical trials regarding pain outcomes are controversial and insufficient to draw conclusions on the effects of KT.

Some limitations should be addressed. Firstly, some control groups might expose significant effects to relieve the symptoms for patients with chronic knee pain, which cannot reflect the virtual effect of the KT group when compared with those control groups. Secondly, some studies were of insufficient quality to warrant data extraction to contrast. Thirdly, we might have excluded relevant but non-English studies. Fourth, significant heterogeneity was encountered perhaps due to various regimens, doses, duration, center settings, populations enrolled, etc., calling for cautious interpretation of the results. Fifth, many of the studies suffer from significant sources of bias. Sixth, the effect in many occasions was assessed by very few studies; thus, the evidence to support it is low. Finally, the PROSPRO registration code was not provided. 
Remarkably, it should be noted that the application of KT was initially designed for patients with chronic knee pain in the early stage [30]. But we found that the long-term effects of Kinesio taping are not significant and outstanding.

\section{Conclusion}

$\mathrm{KT}$ is essential to relieve chronic knee pain and prevent massive use injuries in patients with chronic knee pain but not in a long-term effect. Therefore, KT could be temporarily used in practice for exercise or rehabilitation training.

\section{Data Availability}

No data were used to support this study.

\section{Conflicts of Interest}

The authors declare no conflicts of interest in preparing this article.

\section{References}

[1] P. G. Gerbino, E. D. Griffin, P. A. d'Hemecourt et al., "Patellofemoral pain syndrome," The Clinical Journal of Pain, vol. 22, no. 2, pp. 154-159, 2006.

[2] G. K. Fitzgerald, R. S. Hinman, J. Zeni, M. A. Risberg, L. Snyder-Mackler, and K. L. Bennell, "OARSI clinical trials recommendations: design and conduct of clinical trials of rehabilitation interventions for osteoarthritis," Osteoarthritis and Cartilage, vol. 23, no. 5, pp. 803-814, 2015.

[3] R. A. Dutton, M. J. Khadavi, and M. Fredericson, "Patellofemoral pain," Physical Medicine and Rehabilitation Clinics of North America, vol. 27, no. 1, pp. 31-52, 2016.

[4] S. J. Warden, R. S. Hinman, M. A. Watson, K. G. Avin, A. E. Bialocerkowski, and K. M. Crossley, "Patellar taping and bracing for the treatment of chronic knee pain: a systematic review and meta-analysis," Arthritis \& Rheumatism, vol. 59, no. 1, pp. 73-83, 2008.

[5] M. Denti, D. Tornese, G. Melegati, H. Schonhuber, A. Quaglia, and P. Volpi, "Combined chronic anterior cruciate ligament and posterior cruciate ligament reconstruction: functional and clinical results," Knee Surgery, Sports Traumatology, Arthroscopy, vol. 23, no. 10, pp. 2853-2858, 2015.

[6] K. Rowicki, J. Płomiński, and A. Bachta, "Evaluation of the effectiveness of platelet rich plasma in treatment of chronic pes anserinus pain syndrome," Ortopedia Traumatologia Rehabilitacja, vol. 16, no. 3, pp. 307-318, 2014.

[7] S. K. Poddar and L. Widstrom, "Nonoperative options for management of articular cartilage disease," Clinics in Sports Medicine, vol. 36, no. 3, pp. 447-456, 2017.

[8] M. Morvaridzadeh, S. Fazelian, S. Agah et al., "Effect of ginger (zingiber officinale) on inflammatory markers: a systematic review and meta-analysis of randomized controlled trials," Cytokine, vol. 135, Article ID 155224, 2020.

[9] J. Heshmati, F. Golab, M. Morvaridzadeh et al., "The effects of curcumin supplementation on oxidative stress, sirtuin-1 and peroxisome proliferator activated receptor $\gamma$ coactivator $1 \alpha$ gene expression in polycystic ovarian syndrome (PCOS) patients: a randomized placebo-controlled clinical trial," Diabetes \& Metabolic Syndrome: Clinical Research \& Reviews, vol. 14, no. 2, pp. 77-82, 2020.
[10] S. J. Kamper and N. Henschke, "Kinesio taping for sports injuries," British Journal of Sports Medicine, vol. 47, no. 17, pp. 1128-1129, 2013.

[11] M. E. Kaya, "Does kinesio taping of the knee improve pain and functionality in patients with knee osteoarthritis?: a randomized controlled clinical trial," American Journal of Physical Medicine \& Rehabilitation, vol. 96, no. 1, pp. 25-33, 2017.

[12] H.-y. Cho, E.-H. Kim, J. Kim, and Y. W. Yoon, "Kinesio taping improves pain, range of motion, and proprioception in older patients with knee osteoarthritis," American Journal of Physical Medicine \& Rehabilitation, vol. 94, no. 3, pp. 192-200, 2015.

[13] M. C.-E. Chan, J. W.-J. Wee, and M.-H. Lim, "Does kinesiology taping improve the early postoperative outcomes in anterior cruciate ligament reconstruction? a randomized controlled study," Clinical Journal of Sport Medicine, vol. 27, no. 3, pp. 260-265, 2017.

[14] F. Kocyigit, M. B. Turkmen, M. Acar et al., "Kinesio taping or sham taping in knee osteoarthritis? a randomized, doubleblind, sham-controlled trial," Complementary Therapies in Clinical Practice, vol. 21, no. 4, pp. 262-267, 2015.

[15] E. Akbas, A. O. Atay, and I. Yuksel, "The effects of additional kinesio taping over exercise in the treatment of patellofemoral pain syndrome," Acta Orthop Traumatol Turc, vol. 45, no. 5, pp. 335-341, 2011.

[16] O. Aydoğdu, "Clinical outcomes of kinesio taping applied in patients with knee osteoarthritis: a randomized controlled trial," Journal of Back and Musculoskeletal Rehabilitation, vol. 30, no. 5, pp. 1-7, 2017.

[17] B. Wageck, G. S. Nunes, N. B. Bohlen, G. M. Santos, and M. de Noronha, "Kinesio taping does not improve the symptoms or function of older people with knee osteoarthritis: a randomised trial," Journal of Physiotherapy, vol. 62, no. 3, pp. 153-158, 2016.

[18] K. Homayouni, S. Foruzi, and F. Kalhori, "Effects of kinesiotaping versus non-steroidal anti-inflammatory drugs and physical therapy for treatment of pes anserinus tendinobursitis: a randomized comparative clinical trial," The Physician and Sportsmedicine, vol. 44, no. 3, pp. 252-256, 2016.

[19] K. Lee, C.-W. Yi, and S. Lee, "The effects of kinesiology taping therapy on degenerative knee arthritis patients' pain, function, and joint range of motion," Journal of Physical Therapy Science, vol. 28, no. 1, pp. 63-66, 2016.

[20] T. Kuru, A. Yaliman, and E. E. Dereli, "Comparison of efficiency of Kinesio taping and electrical stimulation in patients with patellofemoral pain syndrome," Acta Orthopaedica et Traumatologica Turcica, vol. 46, no. 5, pp. 385-392, 2012.

[21] K.-Y. Ho, R. Epstein, R. Garcia, N. Riley, and S.-P. Lee, "Effects of patellofemoral taping on patellofemoral joint alignment and contact area during weight bearing," Journal of Orthopaedic \& Sports Physical Therapy, vol. 47, no. 2, pp. 115-123, 2017.

[22] D. W. Edmonds, J. McConnell, J. R. Ebert, T. R. Ackland, and C. J. Donnelly, "Biomechanical, neuromuscular and knee pain effects following therapeutic knee taping among patients with knee osteoarthritis during walking gait," Clinical Biomechanics, vol. 39, pp. 38-43, 2016.

[23] A. Hickey, D. Hopper, T. Hall, and C. Y. Wild, "The effect of the mulligan knee taping technique on patellofemoral pain and lower limb biomechanics," The American Journal of Sports Medicine, vol. 44, no. 5, pp. 1179-1185, 2016.

[24] S. A. Campbell and A. R. Valier, "The effect of kinesio taping on anterior knee pain consistent with patellofemoral pain 
syndrome: a critically appraised topic," Journal of Sport Rehabilitation, vol. 25, no. 3, pp. 288-293, 2016.

[25] S.-E. Lee and S.-H. Cho, "The effect of McConnell taping on vastus medialis and lateralis activity during squatting in adults with patellofemoral pain syndrome," Journal of Exercise Rehabilitation, vol. 9, no. 2, pp. 326-330, 2013.

[26] M. Campolo, J Babu, K Dmochowska, S Scariah, and J Varughese, "A comparison of two taping techniques (kinesio and mcconnell) and their effect on anterior knee pain during functional activities," International Journal of Sports Physical Therapy, vol. 8, no. 2, pp. 105-110, 2013.

[27] D. Moher, "Preferred reporting items for systematic reviews and meta-analyses: the PRISMA statement," PLoS Medicine, vol. 6, no. 7, Article ID e1000097, 2009.

[28] T.-C. Fu, A. M. K. Wong, Y.-C. Pei, K. P. Wu, S.-W. Chou, and Y.-C. Lin, "Effect of Kinesio taping on muscle strength in athletes-a pilot study," Journal of Science and Medicine in Sport, vol. 11, no. 2, pp. 198-201, 2008.

[29] P. L. Chen, "Biomechanics effects of kinesio taping for persons with patellofemoral pain syndrome during stair climbing," in 4th Kuala Lumpur International Conference on Biomedical Engineering 2008: BIOMED 2008 25-28 June 2008 Kuala Lumpur, Malaysia, N. A. Abu Osman and N. A. Abu Osman, Eds., pp. 395-397, Springer Berlin Heidelberg, Berlin, Heidelberg, 2008.

[30] Y.-H. Park and J.-H. Lee, "Effects of proprioceptive sensebased Kinesio taping on walking imbalance," Journal of Physical Therapy Science, vol. 28, no. 11, pp. 3060-3062, 2016. 